\title{
BMJ Open Comparison of the outcomes of warfarin therapy and economics by online and offline anticoagulation management models: protocol for a randomised controlled trial
}

\author{
Xiaotong Xia, ${ }^{1,2}$ Jinglan Fu, ${ }^{1,2}$ Tingting Wu, ${ }^{1,2}$ Wenjun Chen, ${ }^{1,2}$ Zhang Jinhua ${ }^{\odot, 2}$
}

To cite: Xia X, Fu J, Wu T, et al. Comparison of the outcomes of warfarin therapy and economics by online and offline anticoagulation management models: protocol for a randomised controlled trial. BMJ Open 2019;9:e031730. doi:10.1136/ bmjopen-2019-031730

- Prepublication history for this paper is available online. To view these files, please visit the journal online (http://dx.doi org/10.1136/bmjopen-2019031730).

Received 16 May 2019 Revised 06 July 2019 Accepted 01 August 2019

\begin{abstract}
Introduction Warfarin is widely used in the world as oral anticoagulant, but it is difficult to manage patients after medication due to its narrow treatment window and individualised differences. Therefore, every region uses network means to carry out online anticoagulant therapy services. The purpose of this paper is to compare monitoring results and randomised controlled studies of the complications of warfarin treated by offline or online management in a Chinese population.

Methods and analysis This is a randomised controlled, multicentre clinical trial. Taking the Union Hospital Affiliated to Fujian Medical University as the main centre, a randomised controlled study of several subcentres around China produced a nationally representative sample. 496 participants who took warfarin will be recruited and then randomly divided into two groups at a ratio of 1:1. We will collect data on patient characteristics, diagnosis, treatment, hospitalisation results and later complications. Ethics and dissemination The study was approved by the Ethics Committee of the Union Hospital Affiliated to Fujian Medical University. All cooperative hospitals have been approved by the Central Ethics Committee. The results of the survey will be disseminated in future peer review documents and will provide the basis for a management model for patients in China taking warfarin. Trial registration number ChiCTR1900021920.
\end{abstract}

\section{INTRODUCTION}

Warfarin, as the most commonly used oral anticoagulant in clinics, has been used for half a century. Warfarin is widely used in the treatment of cardiovascular diseases such as cardiac valvular disease, cardiac valve replacement, atrial fibrillation, deep venous thrombosis and pulmonary embolism. However, the drug is characterised by a narrow range of effective blood concentrations, complex factors affecting anticoagulant effect and obvious individual differences in efficacy, making it difficult for clinicians to master the dosage of warfarin in the implementation of anticoagulant therapy. The effectiveness
Strengths and limitations of this study

- We are performing a trial to provide new management models, especially for patients living in rural areas or with mobility difficulties.

- Medical records were centrally abstracted in a standard data dictionary and governed by rigorous data quality standards.

- Blinding was not possible with respect to participants.

and safety of long-term warfarin anticoagulant therapy have also become the main factors affecting the quality of life of patients after operation. ${ }^{1}$ Therefore, it is necessary to closely monitor the international normalised ratio (INR) for a long time to adjust the dosage of warfarin in order to achieve both effective prevention and treatment of thrombosis, but also to reduce the occurrence of adverse reactions. Therefore, the long-term management of warfarin after discharge has become particularly important.

The standard warfarin treatment (INR range of 2.0-3.0) is associated with major haemorrhagic rates of up to $5 \%-9 \%$ per year, ${ }^{23}$ while recurrent venous thromboembolism is up to $8 \%$. ${ }^{4}$ The complexity of anticoagulant therapy and the lack of a communication system between the medical staff providing anticoagulant management and the patients increase the risk of poor prognosis. ${ }^{5}$ The incidence of these adverse events depends on the pattern used to manage oral anticoagulant therapy. Therefore, in order to better provide services for patients taking warfarin, many foreign countries have been engaged in early anticoagulant therapy management, such as the establishment of anticoagulant clinic. The use of specialised anticoagulant therapy services reduces the incidence of adverse 
reactions by $50 \%-80 \%$ and medical costs by more than $\$ 1600$ per year compared with conventional management, which bring better outcomes and economic results. ${ }^{6}$

Now most of the world's anticoagulant outpatient medical resources are in advanced central cities, but a larger proportion of patients in need for anticoagulant therapy are living in rural areas. For some patients with mobility difficulties, the extra high cost of travel is a huge economic burden. With the continuous development of social economy and construction of network facilities, using the internet, which provides users with the resource and service to enhance their own economic efficiency, has become the main development trend of the current industries. ${ }^{7}$ With this, online anticoagulant management was developed. Online modes such as telephone follow-up, short message service (SMS), apps, anticoagulant website and other platforms have been used for anticoagulant management for discharge patients and for self-monitoring of patients at home, known as online management mode. Discharged patients at home can communicate with their physicians and pharmacists to adjust the use of drugs and to reduce the risk of adverse events in time.

\section{STUDY DESIGN}

This study is a multicentre, randomised controlled study and will be held from March 2019 to April 2021 with Union Hospital Affiliated to Fujian Medical University as the main centre and with many other medical centres in various regions of China. In order to obtain as many representative samples as possible throughout the country to reflect China's economic development and diversification of medical resources, the characteristics and results of patients throughout the country will be studied in different areas of socioeconomic development. Eventually, we identified seven areas for research distributed geographically, including subcentres in East China, South China, Central China, North China, Northwest China, Southwest Asia and Northeast China (figure 1).

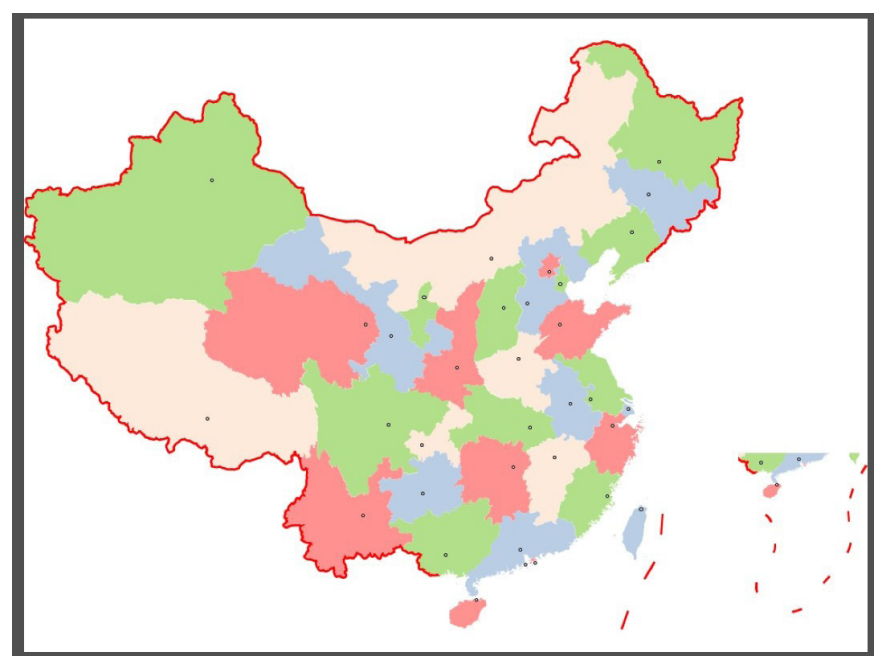

Figure 1 Geographical distribution of subcentres.
The researchers in this study included clinical doctors who have direct contact with patients and who treat them, and clinical pharmacists who provide patients with the safest and most reasonable medication and help doctors make scientific medication options for patients. The research process consists mainly of three parts, namely initial screening, treatment period and follow-up period. In the initial screening, the patient will pass the clinician's physical examination and then included in the assessment. Based on the results of the assessment, patients in the group will sign an informed consent and we will introduce to them all the details of the assessment to make them understood. Once informed consent is obtained, stratified randomised grouping will be performed using a random number generated by the random number table. According to a random 1:1 proportion, patients will be divided into an online group and an offline group. The online group of patients will be discharged through the online anticoagulant management, while the offline group of patients will be discharged to the outpatient clinic for anticoagulant management (figure 2). Both groups of patients will be followed up for 6 months, and the follow-up includes (1) observation indicators: time in therapeutic range (TTR), adverse events and excessive anticoagulant incidence, INR percentage outside the target range, time required to reach treatment INR, dosage adjustment times, and so on; and (2) economic indicators: rehospitalisation fee of warfarin adverse reaction, fare to medical institutions and app registration fee.

\section{STUDY PARTICIPANTS}

The main indications include cardiac valve replacement, atrial fibrillation, pulmonary embolism, venous thrombosis of the lower extremities and so on, which are mainly included in other regional subcentres such as the main centre of Union Hospital Affiliated to Fujian Medical University.

\section{Inclusion criteria}

- Patients in need of warfarin anticoagulant therapy.

- Patients willing to learn to use and accept online or offline warfarin management.

- Patients willing to undergo follow-up.

- Consent of the patient and the signing of an informed consent certificate.

\section{Exclusion criteria}

- Pregnant patients.

- Patients taking or planning to take other oral anticoagulants.

- Patients having experienced therapeutic or subtherapeutic bleeding complications in the past 6 months.

- Patients with expected impending need to interrupt warfarin surgery.

- Patients with severe renal insufficiency (Endogenous creatinine clearance rate, $\mathrm{Ccr} \leq 20 \mathrm{~mL} / \mathrm{min}$ ). 


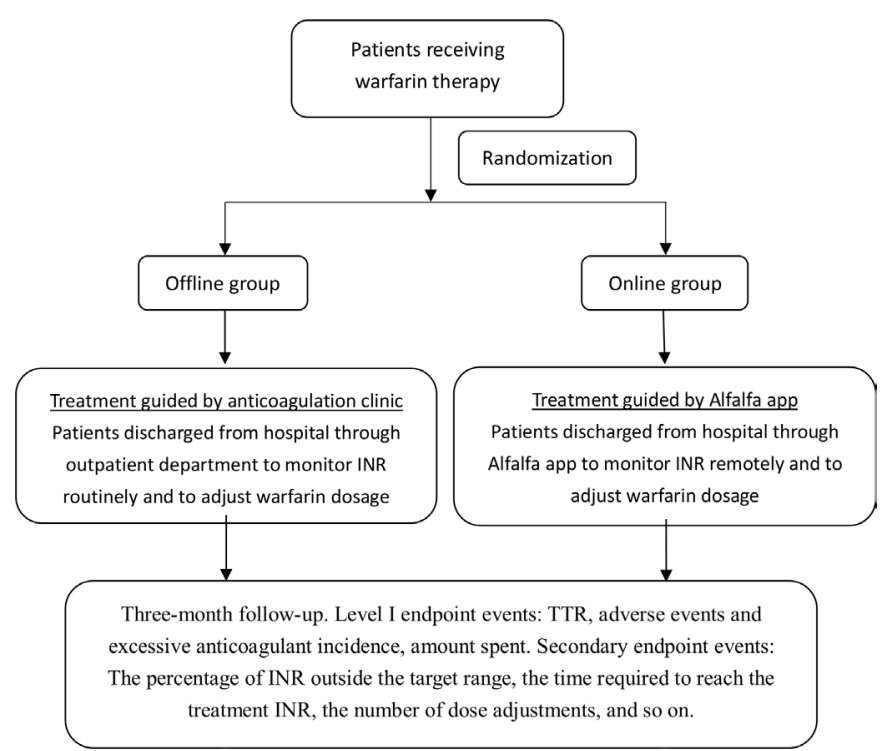

Figure 2 Study flow chart. INR, international normalised ratio. $T T R$, time in therapeutic range.

- Patients with severe hepatic insufficiency (Child-Pugh $\geq 10$ ).

- Patients with severe heart failure (cardiac function New York Heart Association, NYHA grade IV and above).

- Patients with severe infection and respiratory failure.

\section{INTERVENTIONS}

\section{Offline group}

Patients are required to go to the hospital regularly according to medical advice to review the adjustment of coagulation index and warfarin dosage. After patients are enrolled, they will receive routine patient education.

\section{Online groups}

Patients need to pay attention to and download the Alfalfa app (figure 3), and then they will be guided on how to register and use the Alfalfa app, while they learn about relevant anticoagulant knowledge, understand what is

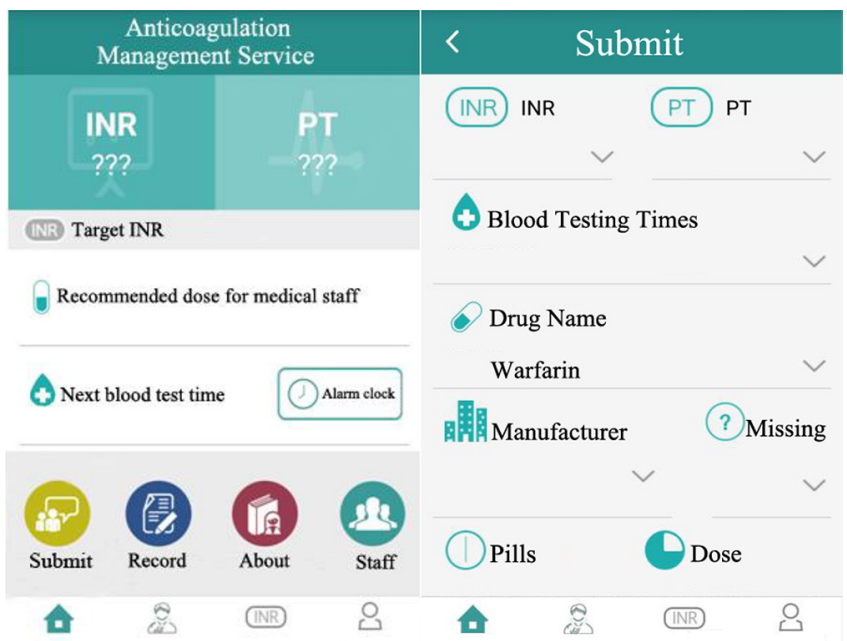

Figure 3 Alfalfa app operation interface for patients. INR, international normalised ratio. PT, Prothrombin Time.
Instructing the patient to download the relevant anticoagulant app after fully understanding the patient's condition.

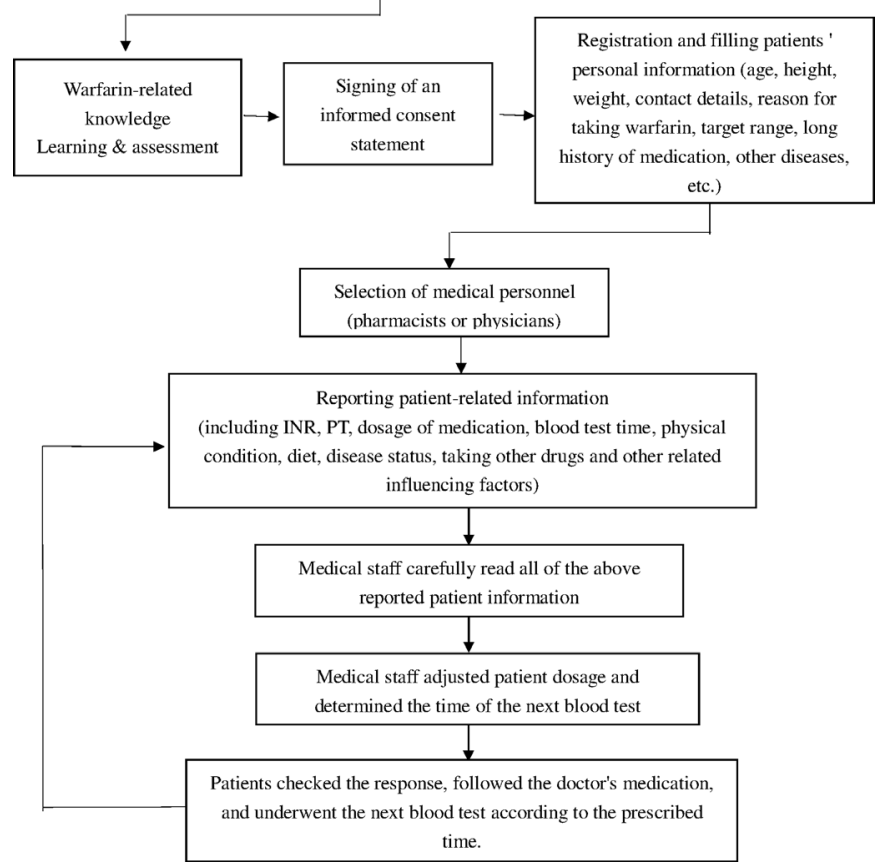

Figure 4 Online reception flow chart. INR, international normalised ratio. PT, Prothrombin Time.

warfarin, why take warfarin, how to take warfarin, what indicators to check when taking warfarin, factors affecting warfarin, what may cause adverse reactions, learn the basic method of warfarin dosage adjustment, monitoring and treatment measures of bleeding during medication, matters needing attention, and so on, and pass the relevant assessment. After having signed the informed consent and registered the user account number, the patient will fill in his personal information, INR target range, other diseases, reasons for taking warfarin and all medicines being taken for long-term use, in order to facilitate the management of the patient's situation for the medical staff in charge later. After successful registration, the patient themselves can choose the medical staff (physician or pharmacist) who will carry out the medication instruction in the future.

Finally, after blood tests in local hospitals, patients can remotely report their current anticoagulant drugs, recent blood tests (INR, international normalised ratio. PT, Prothrombin Time), dosage, blood test time, physical condition, diet, disease status, combined medication and so on, to the designated medical staff through the internet (figure 4). The medical staff carefully read all the patient's reported information and finally develop an individualised anticoagulant programme online to guide patients in adjusting the appropriate dosage, alert relevant considerations and confirm the next blood test time. In addition to reporting blood tests, they can also set medication and blood test alarm alerts-by default, 10 min before taking the drug-and the time to take the 
medication in order to increase patient compliance with the next blood test cycle.

After 6 months of hospital follow-up, data and cost-benefit analyses were carried out through the outcome index and the amount spent during this period (including coagulation index test fee, fare, app registration fee, drug bill and so on).

\section{OUTCOME PARAMETERS AND STATISTICAL ANALYSIS \\ Primary outcome}

- Percentage of time in the target INR range (\%TTR).

- Excessive anticoagulant (defined as INR >4) time ratio.

- Security endpoint event: the occurrence of primary and secondary bleeding events (as level 2 endpoint event). In the light of the International Society on Thrombosis and Haemostasis, the complications of haemorrhage caused by warfarin are classified as major bleeding and secondary haemorrhage. Major bleeding includes fatal haemorrhage, life-threatening bleeding (surgical intervention, need for blood transfusion $\geq 3$ units, irreversible sequelae) and severe bleeding (need for treatment or assessment such as gastric intestinal haemorrhage, joint haematoma, retroperitoneal haemorrhage, fundus haemorrhage, urine, haemoptysis and so on, and need for blood transfusion $\geq 2$ units). Secondary bleeding includes mild nasal haemorrhage, endoscopic haematuria, skin stasis, mild haemorrhoids haemorrhage and so on, and patients do not have serious consequences of bleeding.

- Curative effect endpoint event: the curative effect endpoint event includes the occurrence of thrombosis event, including ischaemic stroke, systemic embolism and transient ischaemic attack, which are confirmed by imaging methods such as colour ultrasound, CT or MRI.

- Economic indicators: warfarin adverse reaction rehospitalisation fee, fare to medical institutions and app registration fee.

\section{Secondary outcomes}

- Extreme INR value.

- The percentage of time above the target INR range.

- The proportion of patients in each group who received a stable dose after the end of follow-up.

\section{SAMPLE SIZE}

Due to the lack of studies on sample size calculation for comparing online and offline anticoagulant management models, we performed the sample calculation based on the results of our previous study and experts' opinion. The TTRs of the online group and the offline group were about $73.1 \%$ and $66 \%{ }^{8}$. If the requirement to grasp is at least $80 \%$, a class of errors will be 0.05 . The study of the test group and control group by 1:1 proportion, calculated through PASS V.11, needs at least 206 patients per group, assuming a dropout rate or loss rate of $20 \%$. Then each group needs at least 248 patients, with a total of 496 patients (using PASS V.11.0 to estimate the sample volume).

\section{DATA COLLECTION}

The researchers are trained. After each hospital collects cases, a unique research identification number for each case is specified. The researchers at the scene collect medical information on all the cases and conceal direct identification information (name, identification number, contact details). ${ }^{9}$ Cases collected in accordance with the selected criteria to take warfarin, according to the random number table stratification, are then divided into the online group and offline group. After the clinical patients are enrolled in the group, all relevant information is recorded, including the demographic information, diagnosis, drug use, test results, 6-month follow-up results and so on. All data are obtained according to standard operation. During the course of the study, the researchers will regularly check the integrity of the subject's records, the accuracy of data entry and the patient's compliance with the research programme, study the progress of the group, and ensure that all operations are carried out in accordance with the programme regulations. A special medical staff will be responsible for the patient's follow-up and INR monitor, and will set up a special schedule. Initially, patients tested their INR once weekly. If the INR was therapeutic for two or three consecutive readings, the interval between tests was increased to a maximum of 1 month. The number of days to reach a stable dose, the INR value and the occurrence of side effects and the time are recorded in detail.

At the beginning of the study, we will provide patients with written instructions and contact phone numbers in case of emergency. During the course of the study, the hypervisor maintains the confidentiality and security of patient information by using data encryption, secure servers and passwords that are set up by both the medical staff and the patients.

\section{PATIENT AND PUBLIC INVOLVEMENT}

Patients or the public were not involved in the design of the study.

\section{STATISTICAL ANALYSES}

The original baseline information and the clinical characteristics of the patients in the test group and the control group are first compared and then the difference of the occurrence of the endpoint events between the two groups. Qualitative data are expressed in percentages, and quantitative data are expressed in mean $\pm \mathrm{SD}(\mathrm{x} \pm \mathrm{S})$ or median and four-digit spacing (IQR). The Kolmogorov-Smirnov method is used to test whether the quantitative data conform to the normal distribution. If so, the 
difference between the two groups of continuous variables is analysed with the application of single factor variance test, otherwise the non-parametric test is adopted. A single factor variance analysis is used to compare the mean of the measured data between samples. The variance of the metering data needs to be tested before variance analysis, for that the variance analysis will be carried out only if variances are equal, or the non-parametric rank and test will be used. The minimum significant difference method is used to compare the mean number between multiple samples one to one. The composition of the sample interstitial rate or genotype are tested under the column table data $\chi^{2}$. Non-paired card square test or the Fisher's exact probability method is applied to test the difference of test rate or composition ratio. We use the Kaplan-Meier curve to indicate the time it takes to reach the treatment target INR for the first time and the time it takes to start from treatment to a stable dose. In this paper, the difference of end event incidence between the experimental group and the control group is tested, and the influence of each factor on endpoint event is analysed by Cox risk regression model. The significance of bilateral examination is statistically significant, and the difference is statistically significant with $\mathrm{p}<0.05$. All statistical analyses are done using IBM SPSS V.22.0 software.

\section{DATA MANAGEMENT}

All physicians, assessors and research assistants will attend training workshops before the conduct of the trial. Investigators in different centres will all be required to follow the standard operating procedures. All study data will be managed as detailed in the full trial protocol and in accordance with the data management plan. The data collection will include all information in the forms. Data will be entered using the double entry method. To ensure data quality and data consistency between the source data and the data entered into the database, two research assistants will independently input the data from the Case Report Form (CRFs) into the database.

\section{ASSESSMENT COSTS}

The method is similar to the clinical results. We will analyse the costs in both groups, showing the total differences between the two groups during follow-up, as well as the relative costs per patient. The economic costs of drugs include direct costs, indirect costs and hidden costs. ${ }^{10}$ Hidden costs include costs caused by adverse drug reactions and so on. Indirect costs include the cost of travel for each patient in order to be tested in a medical institution, and the cost of blood pumping, laboratory inspection fees and other direct costs. The selected medical records are basically the same. Therefore, this study compares the total cost of pharmaceutical drugs, the costs caused by adverse reactions and the fare. This study will use the cost-effect analysis method to find the best solution. The cost-effect ratio is the ratio of the total cost to efficiency.
The smaller it is, the lower the cost to achieve the best results, and the more reasonable the scheme will be.

\section{DISCUSSION}

The study is a rigorous multicentre, randomised controlled study to generate a representative sample of Chinese hospitals and patients who have been taking warfarin for a long time. The study has attracted the active participation of hospitals in various regions of China, ensuring the representativeness of the study population as well as the extensive dissemination of research results, and improving the quality of research. The study may produce one of the larger reports on taking warfarin anticoagulant management in China, and will describe new warfarin management model as well as short-term patient outcomes. Investigating a warfarin management model may reveal the gap between current clinical guidelines and reality, highlighting the need for future improvement efforts to ensure that all patients have better treatment. Exploring new management models, especially for patients living in rural areas or with mobility difficulties, will indicate direction of future management.

This research also has some limitations. First of all, the results depend on the integrity and accuracy of the data. The common problem is that it is easy to miss bleeding, thrombosis and other complications, while the records of laboratory results and imaging tests are very reliable. Therefore, recording adverse reactions will become an important goal of quality improvement. Second, patients' compliance is a key factor. Patients' age, knowledge level and the importance of the disease have an impact on their compliance with the drug, which also has a greater impact on the occurrence of adverse reactions, the time to meet the standards and other indicators. Finally, our research can be internationalised.

The study was the first to be conducted on a nationally representative sample of patients taking warfarin, and assessed the characteristics of Chinese patients, hospitals and regions, as well as the final efficacy indicators and adverse reactions. It provides a basis through which medical personnel and patients can explore the postoperative management model of warfarin that is more suitable for the Chinese population.

\section{ETHICS AND DISSEMINATION}

Eligible clinical patients may enter this study after signing an informed consent letter approved by the Ethics Committee of the Research Hospital, or if the subject does not have the ability to sign the person's legal guardian will need to sign an informed consent in lieu of the patient. The researcher should explain the study at the greatest extent possible to the participants in the group or their guardians.

Any official publication or publication of this research data will protect the privacy of clinical patients, and the identity of the subjects will not be disclosed. The results of 
laboratory tests, other examination results and so on are published for scientific purposes only without disclosing the identity of the subjects.

Contributors ZJ, XX and JF drafted the manuscript. ZJ participated in the design of the study. ZJ, XX, TW, WC and JF coordinated the study. All authors participated, read and approved the final manuscript.

Funding This study was supported by a grant from the Natural Science Foundation of Fujian Province of China (2018Y0037).

Map disclaimer The depiction of boundaries on the map(s) in this article do not imply the expression of any opinion whatsoever on the part of BMJ (or any member of its group) concerning the legal status of any country, territory, jurisdiction or area or of its authorities. The map(s) are provided without any warranty of any kind, either express or implied.

Competing interests None declared.

Patient consent for publication Not required.

Ethics approval The pilot scheme was approved by the Ethics Committee of the Union Hospital Affiliated to Fujian Medical University (Ethics: 2019KY020). The design, implementation and reporting of this clinical study should be in accordance with the ethical principles of the Helsinki Declaration.

Provenance and peer review Not commissioned; externally peer reviewed.

Open access This is an open access article distributed in accordance with the Creative Commons Attribution Non Commercial (CC BY-NC 4.0) license, which permits others to distribute, remix, adapt, build upon this work non-commercially, and license their derivative works on different terms, provided the original work is properly cited, appropriate credit is given, any changes made indicated, and the use is non-commercial. See: http://creativecommons.org/licenses/by-nc/4.0/.

\section{REFERENCES}

1. Jun LIU, Yan-hong ZHU, Jia-jie L, et al. Participation of clinical pharmacists in individualized warfarin anticoagulant therapy based on pharmacogenomics in patients after cardiac valve replacement. Chinese Journal of Hospital Pharmacy 2015;35:249-53.

2. Beyth RJ, Quinn L, Landefeld CS. A multicomponent intervention to prevent major bleeding complications in older patients receiving warfarin. A randomized, controlled trial. Ann Intern Med 2000;133:687-95

3. Finn SD, Callahan CM, Martin DC, et al. The risk for and severity of bleeding complications in elderly patients treated with warfarin. The National Consortium of anticoagulation clinics. Ann Intern Med 1996;124:970-9.

4. Gitter MJ, Jaeger TM, Petterson TM, et al. Bleeding and thromboembolism during anticoagulant therapy: a population-based study in Rochester, Minnesota. Mayo Clin Proc 1995;70:725-33.

5. Staresinic AG, Sorkness CA, Goodman BM, et al. Comparison of outcomes using 2 delivery models of anticoagulation care. Arch Intern Med 2006;166:997.

6. Chiquette E, Amato MG, Bussey HI. Comparison of an anticoagulation clinic with usual medical care: anticoagulation control, patient outcomes, and health care costs. Arch Intern Med 1998;158:1641-7.

7. Zhang B, Sun Z, Ju M. Research progress of telemedicine based on internet of things cloud computing technology in the management of chronic diseases in the elderly. Chinese Journal of Gerontology 2017;37:2835-8.

8. Zhang J, Liu M, Chen Q, et al. Outcomes of an online pharmacistmanaged anticoagulation clinic for individuals on warfarin therapy living in rural communities. Thromb Res 2017;157:136-8.

9. Yu Y, Zhang H, Li X, et al. The China patient-centered Evaluative assessment of cardiac events (China peace) retrospective heart failure study design. BMJ Open 2018;8:e020918.

10. Xu D. Pharmacoeconomics and its analysis. Chinese Journal Of New Drugs And Clinical Remedies 2000;19. 